\title{
Max-weight scheduling across multiple timescales
}

\author{
Mark S. Squillante ${ }^{\text {a }}$, Peter M. van de Ven ${ }^{\text {b,* }}$ \\ a Mathematical Sciences Department, IBM Research, United States \\ b Centrum Wiskunde \& Informatica (CWI), Netherlands
}

\section{A R T I C L E I N F O}

\section{Article history:}

Received 2 January 2017

Received in revised form 1 September 2017

Accepted 30 January 2018

Available online 7 February 2018

\section{Keywords:}

Multiple timescale systems

Hybrid networks

Markov processes

Scheduling

Stability

\begin{abstract}
A B S T R A C T
Many systems consist of a mixture of various resource types that together support better performance relative to those with a single resource type. One important characteristic of these systems is the fact that the various comprising resource types can operate on different timescales, implying that the corresponding control decisions are not made simultaneously. To address the resulting scheduling problem, we present and analyze two variants of max-weight scheduling that are designed to deal with the different timescales of such systems.
\end{abstract}

(C) 2018 Elsevier B.V. All rights reserved.

\section{Introduction}

Present-day systems increasingly include adaptive infrastructures that can involve multiple types of resources. Examples include dual-sourcing inventory systems [14] (e.g., one supplier faster than another supplier), communication networks with noisy channels [8] (e.g., time variations in sources and channels), workforce management [2] (e.g., more experienced employees faster than less experienced employees), smart power grids [7] (e.g., time variations in load and generation with renewable energy), wireless communication with energy harvesting devices [15] (e.g., time variations in wireless transmission and energy harvesting), and hybrid communication networks [1]. In the latter case of hybrid communication networks, which served as the original motivation for the present study, we observe that present-day communication networks increasingly consist of an adaptive infrastructure that may encompass multiple instances of mobile networks (e.g., wireless and cellular networks, public Internet, and various private intranets) together with a wide range of communication technologies (e.g., 3G and 4G cellular, wired and satellite networks) [9]. Many of these systems exploit and interoperate with a diversity of resource types in order to increase both capacity and robustness.

One overlooked property in the literature relevant to systems involving multiple types of resources concerns the operation of the comprising resource types at different timescales. To address this and related issues, there is an important need for mathematical frameworks that support the design and adaptive control of these

\footnotetext{
* Corresponding author.

E-mail address: ven@cwi.nl (P.M. van de Ven).
}

systems. Such a framework includes fundamental performance limits and how these limits impact the design of adaptive control algorithms, convergence of these algorithms in a multiple timescale environment, and assurance of good performance under these algorithms. Our present study seeks to establish fundamental properties of adaptive control policies for the optimal scheduling across various resource types operating at different timescales that ensures low delays and high throughput.

We consider a general system that includes a mixture of various types of resources that operate at different timescales. To elucidate the exposition of our analysis, we focus on a system environment comprising two resource types with one working at a faster timescale than the other. For example, in the context of hybrid networks, the types of resources could be based on satellite and wireless networks where the former operates at a slower timescale than the latter [6], due to its larger propagation delay and round trip time. We note that our analysis extends in a straightforward manner to support an arbitrary number of resource types working at different timescales.

Given the popularity of the max-weight scheduling algorithm [13] in operations research and related fields, we restrict our attention to this class of adaptive control policies. Our goal is to tailor this scheduling algorithm to system environments with timescale differences and to derive its stability, throughput and delay properties. While these fundamental performance properties of the max-weight scheduling algorithm in single-timescale systems have received a great deal of attention in the research literature (see, e.g., $[5,12]$ ), no previous work to our knowledge has studied the performance properties of max-weight scheduling in a system environment that comprises multiple types of resources operating at different timescales. 


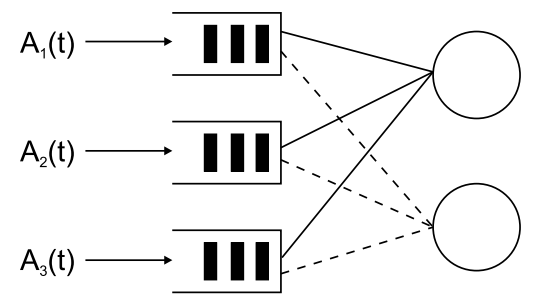

Fig. 1. A stochastic network supporting $N=3$ nodes and two types of server resources (fast and slow).

We speak of a fast resource and a slow resource, where the allocation and feasible service rate over the slow resource remains fixed for a long period of time relative to that of the fast resource. This suggests an interesting new class of scheduling problems in which the longer-term commitment of the slow resource requires more careful scheduling of both resources than would otherwise be the case, possibly taking into account future service on the slow resource when deciding on the usage of the fast resource. Our study, for the first time, explores and models this scheduling problem involving different timescales, and provides initial results on how to handle such environments. We consider a scheduling algorithm that coordinates between the different timescales and compare its delay properties against a scheduling algorithm that does not. To this end we develop new theoretical results that consider the embedded Markov process on transition moments of the slow resource.

In some applications, the problem of scheduling resources across multiple timescales also involves differences in costs among the types of resources, where some resource types are more expensive than others. For example, in a hybrid network with satellite and WiFi resources, a satellite link is typically more costly to operate than a straightforward WiFi link. Although this is outside the scope of the present study, our mathematical framework can be extended to include such issues.

The remainder of this paper is organized as follows. We next present a formal description of our model in Section 2. Section 3 introduces both variants of max-weight scheduling policies considered in our study, and in Section 4 we provide a mathematical analysis of these policies. Section 5 discusses and further explores our results, also considering possible directions for future research.

\section{Mathematical model}

Consider a stochastic network comprising $N$ nodes, indexed by $i=1, \ldots, N$, to which customer traffic arrives over time. Depending on the application, these nodes may for instance represent job classes or wireless devices. The nodes can have customers served across two types of servers that represent diverse types of resources operating at different timescales. This constitutes a stochastic network that consists of a fast server and a slow server, as illustrated in Fig. 1, noting that our results can be extended in a straightforward manner to a general number of types of server resources. Further note that the names fast and slow servers refer to the timescale on which they operate, rather than their service rates.

Time is slotted, indexed by $t=0,1, \ldots$, and each node $i$ maintains a queue of infinite capacity that stores customers awaiting service. Let $Q_{i}(t)$ denote the number of customers waiting at node $i$ at the beginning of slot $t$, and define $\mathbf{Q}(t):=\left(Q_{1}(t), \ldots, Q_{N}(t)\right)$. Let $A_{i}(t)$ denote the number of customers arriving at node $i$ during slot $t$, where arrivals are assumed to be i.i.d. over time and independent among nodes. Denote by $\lambda_{i}=\mathbb{E}\left[A_{i}(0)\right]$ the arrival rate at node $i$, and define $\lambda:=\left(\lambda_{1}, \ldots, \lambda_{N}\right)$. Server resource conditions are such that only a single node can be served over each server resource within a slot; however, a single node is allowed to be served over both resources simultaneously.

Let $F_{i}(t)$ and $S_{i}(t)$ respectively denote the feasible service rate of node $i$ at time $t$ over the fast and slow server resources, representing the number of customers that can be served if the corresponding server resource is selected in slot $t$. These rates may fluctuate over time, and are independent among nodes and server resources. The feasible service rates of the slow resource remain fixed throughout each cycle of $T \geq 2$ slots, i.e.,

$S_{i}(\lfloor t / T\rfloor)=S_{i}(\lfloor t / T\rfloor+1)=\cdots=S_{i}(\lfloor t / T\rfloor+T-1)$,

while the fast resource service rates $F_{i}(t)$ can vary from slot to slot. The feasible service rates for the fast resource and the slow resource are i.i.d. across slots and cycles, respectively. We denote the finite first and second moments of the fast and slow service rates as $f_{i}=\mathbb{E}\left[F_{i}(0)\right], s_{i}=\mathbb{E}\left[S_{i}(0)\right], f_{i}^{(2)}=\mathbb{E}\left[F_{i}(0)^{2}\right], s_{i}^{(2)}=\mathbb{E}\left[S_{i}(0)^{2}\right]$.

At the beginning of each slot (cycle), the scheduling policy decides what node to assign for service on the fast (slow) resource during that slot (cycle). The objective is to quickly stabilize the system and achieve maximum throughput while maintaining acceptably low queue backlog. Let $\mu_{i}(t)$ and $v_{i}(t)$ denote the realized service rates of node $i$ in slot $t$ over the fast and slow resources, respectively, such that $\mu_{i}(t)=F_{i}(t) \cdot \mathbb{1}_{\text {\{fast resource selects node } i \text { in slot } t \text { \} }}$ and $v_{i}(t)=S_{i}(t) \cdot \mathbb{1}_{\text {\{low resource selects node } i \text { in slot } t \text { subject to }(1)\} \text {. Note }}$ that $\mu_{i}(t)$ and $v_{i}(t)$ represent the control variables of the scheduling policy in slot $t$. Define $\mu(t):=\left(\mu_{1}(t), \ldots, \mu_{N}(t)\right)$ and $\boldsymbol{v}(t):=$ $\left(v_{1}(t), \ldots, v_{N}(t)\right)$. Let $\mathbf{0}$ denote the all-zero vector of length $N, \mathbf{e}_{i}$ the $i$ th unit vector of length $N, \hat{F}(t)=\left\{\mathbf{0}, F_{1}(t) \mathbf{e}_{1}, \ldots, F_{N}(t) \mathbf{e}_{N}\right\}$ and $\hat{S}(t)=\left\{\mathbf{0}, S_{1}(t) \mathbf{e}_{1}, \ldots, S_{N}(t) \mathbf{e}_{N}\right\}$. It is readily seen that $\boldsymbol{\mu}(t) \in \hat{F}(t)$ and $\boldsymbol{v}(t) \in \hat{S}(t)$, and then the individual queues evolve according to

$$
\begin{aligned}
Q_{i}(t+1) & =Q_{i}(t)+A_{i}(t)-\mu_{i}(t)-v_{i}(t)+\kappa_{i}(t), \\
\kappa_{i}(t) & =\left(\mu_{i}(t)+v_{i}(t)-Q_{i}(t)-A_{i}(t)\right)^{+} .
\end{aligned}
$$

\section{Scheduling policy and capacity region}

We present two versions of the max-weight scheduling algorithm that differ in the extent to which the fast and slow resources coordinate. The first algorithm makes scheduling decisions in an uncoordinated manner where each resource independently applies a max-weight scheduling strategy according to its own timescale. Namely, after observing the feasible service rates, the slow and fast resources respectively choose at the beginning of each cycle and slot the vectors $v^{*}$ and $\mu^{*}$ that satisfy

$$
\begin{aligned}
& \boldsymbol{v}^{*}(t)= \begin{cases}\underset{\boldsymbol{v} \in \hat{S}(t)}{\operatorname{argmax}} \sum_{i=1}^{N} Q_{i}(t) v_{i}, & t=0, T, 2 T, \ldots, \\
\boldsymbol{v}^{*}(t-I(t)), & \text { otherwise, }\end{cases} \\
& \boldsymbol{\mu}^{*}(t)=\underset{\boldsymbol{\mu} \in \hat{\mathrm{F}}(t)}{\operatorname{argmax}} \sum_{i=1}^{N} Q_{i}(t) \mu_{i},
\end{aligned}
$$

where $I(t):=t-T\lfloor t / T\rfloor$ represents the number of slots into the cycle associated with time $t$. Thus (4) reflects the fact that the service decision of the slow resource remains fixed for each cycle of $T$ slots. The second policy we consider coordinates the scheduling decisions of the fast resource to take into account the decisions of the slow resource, which continue to be governed by (4), such that

$\boldsymbol{\mu}^{*}(t)=\underset{\boldsymbol{\mu} \in \hat{F}(t)}{\operatorname{argmax}} \sum_{i=1}^{N} \mu_{i}(t)\left[Q_{i}(t)-(T-I(t)) v_{i}^{*}(t)\right]$.

In (6) we take into account the fact that the slow resource will continue to serve the same node until the end of the cycle. 
Before turning to our main results, we consider the stochastic network capacity region which is defined to be the closure of the set of all arrival rate vectors $\lambda$ that can be stably supported by the network, considering all possible scheduling decisions. Recall that the feasible service rates are i.i.d. and denote $\pi(f, s)=$ $\mathbb{P}[(F(t), S(t))=(f, s)]$. Known results in the literature characterize the capacity region and its coverage by randomized policies (see, e.g., [5]), as summarized in the following lemma.

Lemma 1. The capacity region $\mathcal{C}$ is given by the interior of

$\left\{\lambda \in \mathbb{R}_{+}^{N} \mid \exists(\boldsymbol{\mu}(u), \boldsymbol{v}(u)) \in \hat{F}(t) \times \hat{S}(t):\right.$

$$
\left.\lambda=\lim _{t \rightarrow \infty} \frac{1}{t} \sum_{u=0}^{t-1} \boldsymbol{\mu}(u)+\boldsymbol{v}(u)\right\},
$$

and, for any $\lambda \in \mathcal{C}$, there exists a distribution $\sigma_{(f, s)}$ such that

$\lambda=\sum_{(f, s)} \pi(f, s) \sum_{(\boldsymbol{\mu}(t), \boldsymbol{v}(t)) \in \hat{F}(t) \times \hat{S}(t)}(\boldsymbol{\mu}+\boldsymbol{v}) \sigma_{(f, s)}(\boldsymbol{\mu}, \boldsymbol{v})$.

\section{Main results}

We now consider the stochastic process $\mathbf{X}(t)=(\mathbf{Q}(t), I(t))$ that tracks over time the per-node backlog and cycle stage, with the goal of deriving the stability region of $\mathbf{X}(t)$ and bounds on the stationary average backlog. Observe that $\mathbf{X}(t)$ is not Markovian, since the allocation and feasible service rate of the slow resource is fully determined by the state of the system at the beginning of the cycle. Owing to the fact that $\mathbf{X}(t)$ is not Markovian, we cannot readily apply in a straightforward manner existing results on the stability region and queue-backlog bounds (such as those found in [5]). Instead we must demonstrate stability by first considering the process embedded on cycle renewal instances, from which we will derive the stability of the general process and its associated queue-length bounds. Let $\|\mathbf{Q}(t)\|_{1}$ denote the 1-norm of the $\mathbf{Q}(t)$ associated with $\mathbf{X}(t)$. The following lemma provides sufficient stability conditions and a queue-length bound for a system operating across multiple time-scales. We note that both the proof and the bound are different from similar results on single time-scale systems such as Lemma 4.1 in [5].

Lemma 2. If there exist a non-negative function $L(\cdot)$ and positive constants $B, \epsilon$ such that, for all $k \in \mathbb{Z}_{+}$and all $(\mathbf{q}, \tau) \in\left(\mathbb{Z}_{+}^{N} \times\right.$ $\{0,1 \ldots, T-1\})$,

$\mathbb{E}[L(\mathbf{X}((k+1) T))-L(\mathbf{X}(k T)) \mid \mathbf{X}(k T)=(\mathbf{q}, \tau)] \leq B-\epsilon\|\mathbf{q}\|_{1}$,

then $\mathbf{X}(t)$ is stable and

$\lim _{t \rightarrow \infty} \frac{1}{t} \sum_{u=0}^{t-1} \mathbb{E}\left[\|\mathbf{Q}(u)\|_{1}\right] \leq \frac{B}{\epsilon}+\|\lambda\|_{1} T$.

Proof. First, we show that for the embedded chain $\{\mathbf{X}(k T)\}_{k \geq 0}$

$\limsup _{l \rightarrow \infty} \frac{1}{l} \sum_{k=0}^{l-1} \mathbb{E}\left[\|\mathbf{Q}(k T)\|_{1}\right] \leq \frac{B}{\epsilon}$.

Consider the conditional drift of the embedded process given in supposition (7). By conditioning on the value of $\mathbf{x}=(\mathbf{q}, \tau)$, starting from some initial state $\mathbf{X}(0)=\mathbf{x}_{0}$, we obtain

$$
\begin{aligned}
& \sum_{\mathbf{x}} \mathbb{P}\left[\mathbf{X}(k T)=\mathbf{x} \mid \mathbf{X}(0)=\mathbf{x}_{0}\right] \mathbb{E}[L(\mathbf{X}(k T+1))-L(\mathbf{X}(k T)) \mid \\
& \mathbf{X}(k T)=\mathbf{x}] \leq \sum_{\mathbf{x}} \mathbb{P}\left[\mathbf{X}(k T)=\mathbf{x} \mid \mathbf{X}(0)=\mathbf{x}_{0}\right]\left(B-\epsilon\|\mathbf{X}\|_{1}\right),
\end{aligned}
$$

which renders

$\mathbb{E}_{\mathbf{x}_{0}}[L(\mathbf{X}((k+1) T))]-\mathbb{E}_{\mathbf{x}_{0}}[L(\mathbf{X}(k T))] \leq \mathbb{E}_{\mathbf{x}_{0}}\left[B-\epsilon\|\mathbf{Q}(k T)\|_{1}\right]$.

Upon summing both sides over $k=0, \ldots, l-1, l \in \mathbb{Z}^{+}$, we have

$-\mathbb{E}_{\mathbf{x}_{0}}[L(\mathbf{X}(0))]+\mathbb{E}_{\mathbf{x}_{0}}[L(\mathbf{X}(l T))] \leq l B-\epsilon \sum_{k=0}^{l-1} \mathbb{E}_{\mathbf{x}_{0}}\left[\|\mathbf{Q}(k T)\|_{1}\right]$.

Dividing both sides by $l$ and $\epsilon$, and rearranging terms yields

$\frac{1}{l} \sum_{k=0}^{l-1} \mathbb{E}_{\mathbf{x}_{0}}\left[\|\mathbf{Q}(k T)\|_{1}\right] \leq \frac{B}{\epsilon}+\frac{1}{l \epsilon}\left(\mathbf{x}_{0}-\mathbb{E}_{\mathbf{x}_{0}}[L(\mathbf{X}(l T))]\right)$.

By Foster's criterion we know that $\frac{1}{l} \mathbb{E}_{\mathbf{x}_{0}}[L(\mathbf{X}(l T))] \downarrow 0$ as $l \rightarrow \infty$, and thus we obtain the desired result in (9).

Next, we demonstrate the stability of $\mathbf{X}(t)$, assuming for contradiction the opposite to be true. Then for any finite set $\mathbb{S}$ and any starting state $\mathbf{x}_{0}$, we have $\lim _{t \rightarrow \infty} \mathbb{P}[\mathbf{X}(t) \in \mathbb{S}] \rightarrow 0$. Now, letting $\mathbb{S}_{M}=\left\{\mathbf{x} \in \mathbb{Z}_{+}^{N} \mid\|\mathbf{q}\|_{1}<M<\infty\right\}$, we derive

$$
\begin{aligned}
\frac{1}{l+1} \sum_{k=0}^{l} \mathbb{E}\left[\|\mathbf{Q}(k T)\|_{1}\right] & \geq \frac{1}{l+1} \sum_{k=0}^{l}(M+1) \mathbb{E}\left[\mathbb{1}_{\left\{\mathbf{X}(k T) \in \mathbb{S}_{M}^{c}\right\}}\right] \\
& =\frac{M+1}{l+1} \sum_{k=0}^{l}\left(1-\mathbb{P}\left[\mathbf{X}(k T) \in \mathbb{S}_{M}\right]\right) .
\end{aligned}
$$

Taking the limit as $l \rightarrow \infty$, the right-hand side converges to $M+1$, thus rendering a contradiction with the existence of the limit (9) since the above inequality holds for any $M>0$.

Finally, in order to establish the bound (8), first observe that

$\mathbb{E}[\mathbf{Q}(k T+t)] \leq\|\lambda\|_{1} t+\mathbb{E}[\mathbf{Q}(k T)], \quad \forall t \geq 0$.

Considering up to time $k T$ and letting $t=k T+1$, we have

$$
\begin{aligned}
\frac{1}{t} \sum_{u=0}^{t-1} \mathbb{E}\left[\|\mathbf{Q}(u)\|_{1}\right] & \leq \frac{1}{k} \sum_{l=0}^{k-1}\left(\mathbb{E}\left[\|\mathbf{Q}(l T)\|_{1}\right]+\|\lambda\|_{1} T\right) \\
& =\frac{1}{k} \sum_{l=0}^{k-1} \mathbb{E}\left[\|\mathbf{Q}(l T)\|_{1}\right]+\|\lambda\|_{1} T .
\end{aligned}
$$

Taking $k, t \rightarrow \infty$ as $t=k T+1$, then (8) follows from (9).

Throughout the remainder of this section, let $\rho$ denote the workload of the system, which is a solution to the equation

$1-\rho=\max _{\epsilon>0} \lambda+\epsilon \in \mathcal{C}$.

\subsection{Uncoordinated scheduling policy}

Before stating our main results for the uncoordinated policy in (4) and (5), we need the following auxiliary lemma. Define $\epsilon:=$ $(\epsilon, \ldots, \epsilon), Z_{i}(t):=\left(A_{i}(t)-\mu_{i}^{*}(t)-v_{i}^{*}(t)+\kappa_{i}^{*}(t)\right), \gamma:=\lambda_{i}+1-\rho$, and $C(t, s):=\theta_{1}^{C}(t, s)$ if $I(t+s)=s, C(t, s):=\theta_{1}^{C}(t, s)+\theta_{2}^{C}(t, s)$ if $I(t+s)>s$ and $C(t, s):=\theta_{1}^{C}(t, s)+\theta_{3}^{C}(t, s)$ if $I(t+s)<s$, where

$$
\begin{aligned}
\theta_{1}^{C}(t, s)= & (s+1)\left(f_{i}^{(2)}+s_{i}^{(2)}+2 s_{i} f_{i}\right) / 4 \\
& +s\left(f_{i}+2 s_{i}-\lambda_{i}\right)\left(\lambda_{i}+1-\rho\right), \\
\theta_{2}^{C}(t, s)= & (I(t+s)-s) \lambda_{i} \gamma+(I(t+s)-s)\left(f_{i}+s_{i}\right) s_{i}, \\
\theta_{3}^{C}(t, s)= & (s-T(t+s))\left(\lambda_{i}-f_{i}-s_{i}\right) \gamma+(I(t+s)-s) \lambda_{i} s_{i} .
\end{aligned}
$$

Lemma 3. Let $\kappa_{i}^{*}(t)$ be as in (3) with $v_{i}(t)=v_{i}^{*}(t)$ and $\mu_{i}(t)=\mu_{i}^{*}(t)$ as in (4) and (5), respectively. Given the system workload $\rho$, we then have for all $s=0,1, \ldots, T-1$

$$
\sum_{i=1}^{N} \mathbb{E}\left[Q_{i}(t) Z_{i}(t+s) \mid \mathbf{Q}(t)=\mathbf{q}\right] \leq C(t, s)-(1-\rho)\|\mathbf{q}\|_{1} .
$$


Proof. We first bound the individual components of the left-hand side of (11). To start, it is readily verified that

$\mathbb{E}\left[Q_{i}(t) A_{i}(t+s) \mid \mathbf{Q}(t)=\mathbf{q}\right]=q_{i} \lambda_{i}$.

By the definition of $\kappa_{i}^{*}(t)$ in (3), we have

$\mathbb{E}\left[Q_{i}(t) \kappa_{i}^{*}(t+s) \mid \mathbf{Q}(t)=\mathbf{q}\right]=\mathbb{E}\left[Q_{i}(t)\left(Z_{i}(t+s)\right)^{+} \mid \mathbf{Q}(t)=\mathbf{q}\right]$.

Using the fact that

$Q_{i}(t)+\sum_{u=0}^{s-1} V_{i}(t+u) \leq Q_{i}(t+s) \leq Q_{i}(t)+\sum_{u=0}^{s-1} A_{i}(t+u)$,

where $V_{i}(t)=A_{i}(t)-F_{i}(t)-S_{i}(t)$, together with the bounds $\mu_{i}(t+s) \leq F_{i}(t+s)$ and $v_{i}(t+s) \leq S_{i}(t+s)$, we obtain

$$
\begin{aligned}
& \mathbb{E}\left[Q_{i}(t) \kappa_{i}^{*}(t+s) \mid \mathbf{Q}(t)=\mathbf{q}\right] \\
\leq & \mathbb{E}\left[Q _ { i } ( t ) \left\{\sum_{u=0}^{s-1}\left(F_{i}(t+u)+S_{i}(t+u)\right)+F_{i}(t+s)+S_{i}(t+s)\right.\right. \\
& \left.\left.-Q_{i}(t)\right\}^{+} \mid \mathbf{Q}(t)=\mathbf{q}\right] \leq \frac{1}{4}(s+1)\left(f_{i}^{(2)}+s_{i}^{(2)}+2 f_{i} s_{i}\right) .
\end{aligned}
$$

The second inequality follows from the fact that the maximum operator bounds the left-hand side.

To bound the $Q_{i}(t) \mu_{i}^{*}(t+s)$ term, first applying (13) renders

$Q_{i}(t) \mu_{i}^{*}(t+s) \geq\left\{Q_{i}(t+s)-\sum_{u=0}^{s-1} A_{i}(t+u)\right\} \mu_{i}^{*}(t+s)$.

Let $(\tilde{\boldsymbol{\mu}}, \tilde{\boldsymbol{v}})$ denote the result of a randomized policy in Lemma 1 such that $\mathbb{E}\left[\tilde{\mu}_{i}+\tilde{\nu}_{i}\right]=\lambda_{i}+\rho$. Then, by the choice of $\mu_{i}^{*}$ in (5),

$\sum_{i=1}^{N} Q_{i}(t+s) \mu_{i}^{*}(t+s) \geq \sum_{i=1}^{N} Q_{i}(t+s) \mathbb{E}_{\sigma(t+s)}\left[\mu_{i}(t+s)\right]$.

Upon combining (15) and (16), we obtain

$$
\begin{aligned}
\sum_{i=1}^{N} Q_{i}(t) \mu_{i}^{*}(t+s) \geq & \sum_{i=1}^{N} Q_{i}(t+s) \mathbb{E}_{\sigma(t+s)}\left[\mu_{i}(t+s)\right] \\
& -S_{i}(t+s) \sum_{u=0}^{s-1} A_{i}(t+u)
\end{aligned}
$$

Using (13) again, we have

$$
\begin{aligned}
\sum_{i=1}^{N} Q_{i}(t) \mu_{i}^{*}(t+s) \geq \sum_{i=1}^{N} & {\left[\left\{Q_{i}(t)+\sum_{u=0}^{s-1} V_{i}(t+u)\right\} \mathbb{E}_{\sigma(t+s)}\left[\mu_{i}(t+s)\right]\right.} \\
& \left.-S_{i}(t+s) \sum_{u=0}^{s-1} A_{i}(t+u)\right]
\end{aligned}
$$

Next, the term $Q_{i}(t) v_{i}^{*}(t+s)=Q_{i}(t) v_{i}^{*}(t+s-I(t+s))$ can be bounded in a similar fashion. We distinguish these bounds for the three different cases of $I(t+s)$ values.

Case 1: $I(t+s)=s$. Here $v_{i}^{*}(t+s-I(t+s))=v_{i}^{*}(t)$, and thus

$\sum_{i=1}^{N} Q_{i}(t) v_{i}^{*}(t+s-I(t+s)) \geq \sum_{i=1}^{N} Q_{i}(t) \mathbb{E}_{\sigma(t)}\left[\tilde{v}_{i}(t)\right]$.

Case 2: $I(t+s)>s$. Let $\hat{t}=t+s-I(t+s)<t$ denote the time when the slow resource service rate for slot $t+s$ was set. It then follows from (13) that

$$
\begin{aligned}
& \sum_{i=1}^{N} Q_{i}(t) v_{i}^{*}(\hat{t}) \geq \sum_{i=1}^{N}\left[Q_{i}(\hat{t})+\sum_{u=0}^{t-\hat{t}-1} V_{i}(\hat{t}+u)\right] v_{i}^{*}(\hat{t}) \\
\geq & \sum_{i=1}^{N} Q_{i}(\hat{t}) \mathbb{E}_{\sigma(\hat{t})}\left[\tilde{v}_{i}(\hat{t})\right]-\sum_{i=1}^{N} S_{i}(t) \sum_{u=0}^{t-\hat{t}-1}\left(F_{i}(\hat{t}+u)+S_{i}(\hat{t}+u)\right) .
\end{aligned}
$$

Therefore, upon bounding $Q_{i}(\hat{t})$ using (13), we obtain

$$
\begin{aligned}
\sum_{i=1}^{N} Q_{i}(t) v_{i}^{*}(\hat{t}) & \geq \sum_{i=1}^{N}\left\{Q_{i}(t)-\sum_{u=0}^{t-\hat{t}-1} A_{i}(\hat{t}+u)\right\} \mathbb{E}_{\sigma(\hat{t})}\left[\tilde{v}_{i}(\hat{t})\right] \\
& -\sum_{i=1}^{N} S_{i}(t) \sum_{u=0}^{t-\hat{t}-1}\left(F_{i}(\hat{t}+u)+S_{i}(\hat{t}+u)\right)
\end{aligned}
$$

Case 3: $I(t+s)<s$. Here $\hat{t}=t+s-I(t+s)>t$, and thus we use (13) to obtain the bound

$$
\begin{aligned}
\sum_{i=1}^{N} Q_{i}(t) v_{i}^{*}(\hat{t}) & \geq \sum_{i=1}^{N}\left[Q_{i}(\hat{t})-\sum_{u=0}^{\hat{t}-t-1} A_{i}(t+u)\right] v_{i}^{*}(\hat{t}) \\
& \geq \sum_{i=1}^{N}\left[Q_{i}(\hat{t}) \mathbb{E}_{\sigma(\hat{t})}\left[\tilde{v}_{i}(\hat{t})\right]-S_{i}(t) \sum_{u=0}^{\hat{t}-t-1} A_{i}(t+u)\right]
\end{aligned}
$$

Then, using (13) once more, we have

$$
\begin{aligned}
\sum_{i=1}^{N} Q_{i}(t) v_{i}^{*}(\hat{t}) \geq \sum_{i=1}^{N} & {\left[\left\{Q_{i}(t)+\sum_{u=0}^{\hat{t}-t-1} V_{i}(t+u)\right\} \mathbb{E}_{\sigma(\hat{t})}\left[\tilde{v}_{i}(\hat{t})\right]\right.} \\
& \left.-S_{i}(t) \sum_{u=0}^{\hat{t}-t-1} A_{i}(t+u)\right] .
\end{aligned}
$$

Hence, depending on the value of $I(t+s)$, we have three different bounds for $\sum_{i=1}^{N} \mathbb{E}\left[Q_{i}(t) Z_{i}(t+s) \mid \mathbf{Q}(t)=\mathbf{q}\right]$. By substituting (12), (14), (17), (18), (19), (21) for the individual terms, and using the fact that $\mathbb{E}\left[\mathbb{E}_{\sigma(t)}\left[\tilde{\mu}_{i}(t)\right]+\mathbb{E}_{\sigma(t)}\left[\tilde{v}_{i}(t)\right]\right]=\lambda_{i}+(1-\rho)$, we then arrive at (11) for the different instances of $C(t, s)$.

From Lemmas 2 and 3, we now derive the stability region and an upper bound on delay for a stochastic network operating under the uncoordinated scheduling policy in (4) and (5).

Theorem 1. Let $\lambda \in \mathcal{C}$ and suppose that scheduling decisions are made according to (4) and (5). Define $\hat{a}_{i}(s):=\left(\sum_{u=0}^{s-1} A_{i}(t+u)\right)^{2}$. Then the network is stable and the average backlog is upper bounded as

$$
\begin{aligned}
\lim _{t \rightarrow \infty} \frac{1}{t} \sum_{u=0}^{t-1} \mathbb{E}\left[\|\mathbf{Q}(u)\|_{1}\right] \leq & \frac{1}{1-\rho}\left(\frac{1}{2} \sum_{i=1}^{N} \hat{a}_{i}(T)+\sum_{s=0}^{T-1} \theta_{1}^{C}(s, s)\right) \\
& +\|\lambda\|_{1} T .
\end{aligned}
$$

Proof. We use the quadratic Lyapunov function $L(\mathbf{x})=\sum_{i=1}^{N} x_{i}^{2}$ and consider the $T$-slot drift

$\Delta_{T} L(\mathbf{q}, t)=\mathbb{E}[L(\mathbf{Q}(t+T))-L(\mathbf{Q}(t)) \mid \mathbf{Q}(t)=\mathbf{q}]$.

After some calculations, we obtain from (2) and (3) that

$Q_{i}^{2}(t+T) \leq Q_{i}^{2}(t)+2 Q_{i}(t)\left(\sum_{u=0}^{T-1} Z_{i}(t+u)\right)+\hat{a}_{i}(T)$

Substituting this into (23) yields

$\Delta_{T} L(\mathbf{q}, t) \leq \mathbb{E}\left[\sum_{i=1}^{N} 2 Q_{i}(t)\left(\sum_{u=0}^{T-1} Z_{i}(t+u)\right)+\hat{a}_{i}(T) \mid \mathbf{Q}(t)=\mathbf{q}\right]$. 
Next, we use Lemma 3 to further evaluate the upper bound for the drift (24), and determine when the conditions of Lemma 2 are satisfied. To this end, we look for a $B$ and $\epsilon$ such that

$\Delta_{T} L(\mathbf{q}, k T) \leq B-\epsilon\|\mathbf{q}\|_{1}, \quad k=0,1, \ldots$

After some calculations, we see that for all $k \geq 0$

$\Delta_{T} L(\mathbf{q}, k T) \leq \sum_{i=1}^{N} \hat{a}_{i}(T)+2\left(-T\|\mathbf{q}\|_{1}(1-\rho)+\sum_{s=0}^{T-1} \theta_{1}^{C}(s, 0)\right)$,

and then (22) readily follows.

\subsection{Coordinated scheduling policy}

We now obtain analogous results for the coordinated scheduling policy in (4) and (6), starting with the following lemma. Recall that $\epsilon:=(\epsilon, \ldots, \epsilon), Z_{i}(t):=\left(A_{i}(t)-\mu_{i}^{*}(t)-v_{i}^{*}(t)+\kappa_{i}^{*}(t)\right)$ and $\gamma:=\lambda_{i}+1-\rho$, and define $D(t, s)=\theta_{1}^{D}(t, s)$ if $I(t+s)=s, D(t, s)=$ $\theta_{1}^{D}(t, s)+\theta_{2}^{D}(t, s)$ if $I(t+s)>s$ and $D(t, s)=\theta_{1}^{D}(t, s)+\theta_{3}^{D}(t, s)$ if $I(t+s)<s$ where

$\theta_{1}^{D}(t, s):=(s+1)\left(f_{i}^{(2)}+s_{i}^{(2)}+2 s_{i} f_{i}\right) / 4+\left(s\left(f_{i}+2 s_{i}-\lambda_{i}\right)+s_{i} T\right) \gamma$,

$\theta_{2}^{D}(t, s):=(I(t+s)-s) \lambda_{i} \gamma+(I(t+s)-s)\left(f_{i}+s_{i}\right) s_{i}$,

$\theta_{3}^{D}(t, s):=(s-T(t+s))\left(\lambda_{i}-f_{i}-s_{i}\right) \gamma+(I(t+s)-s) \lambda_{i} s_{i}$.

Lemma 4. Let $\kappa_{i}^{*}(t)$ be as in (3) with $v_{i}(t)=v_{i}^{*}(t)$ and $\mu_{i}(t)=\mu_{i}^{*}(t)$ as in (4) and (6), respectively. Given the system workload $\rho$, we then have for all $s=0,1, \ldots, T-1$

$$
\sum_{i=1}^{N} \mathbb{E}\left[Q_{i}(t) Z_{i}(t+s) \mid \mathbf{Q}(t)=\mathbf{q}\right] \leq D(t, s)-(1-\rho)\|\mathbf{q}\|_{1} .
$$

Proof. We again want to bound the individual components of (27) where we can reuse the bounds on $Q_{i}(t) A_{i}(t+s), Q_{i}(t) \kappa_{i}^{*}(t+s)$ and $Q_{i}(t) \nu_{i}^{*}(t+s)$ from the proof of Lemma 3. What remains is to bound $Q_{i}(t) \mu_{i}^{*}(t+s)$. First, according to (13), we observe

$$
\begin{aligned}
Q_{i}(t) \mu_{i}^{*}(t+s) \geq & \left\{Q_{i}(t+s)-(T-I(t+s)) \mathbb{1}_{\left\{v_{i}(t)>0\right\}} s_{i}\right. \\
& \left.-\sum_{u=0}^{s-1} A_{i}(t+u)\right\} \mu_{i}^{*}(t+s) .
\end{aligned}
$$

Using the randomized policy $\tilde{\boldsymbol{\mu}}$ as before, it follows that

$$
\begin{gathered}
\sum_{i=1}^{N}\left[Q_{i}(t) \mu_{i}^{*}(t+s) \geq \sum_{i=1}^{N}\left\{Q_{i}(t+s)-(T-I(t+s)) \mathbb{1}_{\left\{v_{i}(t)>0\right\}} s_{i}\right.\right. \\
\left.\left.-\sum_{u=0}^{s-1} A_{i}(t+u)\right\} \mathbb{E}\left[\tilde{\mu}_{i}(t+s)\right]-S_{i}(t+s) \sum_{u=0}^{s-1} A_{i}(t+u)\right] .
\end{gathered}
$$

Applying (13) one final time, we obtain

$$
\begin{array}{r}
\sum_{i=1}^{N}\left[Q_{i}(t) \mu_{i}^{*}(t+s) \geq \sum_{i=1}^{N}\left\{Q_{i}(t+s)-T s_{i}+\sum_{u=0}^{s-1} V_{i}(t+u)\right.\right. \\
\left.\left.-\sum_{u=0}^{s-1} A_{i}(t+u)\right\} \mathbb{E}\left[\tilde{\mu}_{i}(t+s)\right]-S_{i}(t+s) \sum_{u=0}^{s-1} A_{i}(t+u)\right] .
\end{array}
$$

The result then readily follows by substitution.

Exploiting the above and earlier lemmas, we derive the stability region and an upper bound on delay for stochastic networks under the coordinated scheduling policy in (4) and (6).
Theorem 2. Let $\lambda \in \mathcal{C}$ and suppose that scheduling decisions are made according to (4) and (6). Define $\hat{a}_{i}(s):=\left(\sum_{u=0}^{s-1} A_{i}(t+u)\right)^{2}$. Then the network is stable and the average backlog is upper bounded as

$$
\begin{aligned}
\lim _{t \rightarrow \infty} \frac{1}{t} \sum_{u=0}^{t-1} \mathbb{E}\left[\|\mathbf{Q}(u)\|_{1}\right] \leq & \frac{1}{1-\rho}\left(\frac{1}{2} \sum_{i=1}^{N} \hat{a}_{i}(T)+\sum_{s=0}^{T-1} \theta_{1}^{D}(s, s)\right) \\
& +\|\lambda\|_{1} T .
\end{aligned}
$$

The proof of Theorem 2 is analogous to that of Theorem 1, with $\theta_{i}^{C}(t, s)$ replaced with $\theta_{i}^{D}(t, s), i=1,2,3$, and thus omitted.

\section{Discussion}

We have shown in Theorems 1 and 2 that the uncoordinated and coordinated scheduling policies have the same stability region, but may yield different backlog and delay properties with respect to (22) and (31). The fact that these modified versions of maxweight scheduling remain throughput-optimal is in a sense not very surprising, as similar robustness of the max-weight algorithm has been previously demonstrated in settings with imperfect information [4] and reconfiguration delays [3].

The different backlog behaviors between both policies is probably more interesting. Intuitively, one can imagine that the coordinated policy renders better performance by taking into account within a cycle the future slow resource service allocations when making scheduling decisions for the fast resource. It turns out, however, that the coordinated policy is not necessarily better than the uncoordinated policy, as suggested by the bounds in (22) and (31). More specifically, there can be situations where either policy outperforms the other policy with respect to backlog and delay properties.

To confirm this claim, we simulate a 2-node stochastic network under different parameters and compare the performance of both policies. Fig. 2 plots the percentage of relative improvement of coordinated scheduling over uncoordinated scheduling, in terms of the average queue length of 10 simulation runs each consisting of $510^{5}$ time slots. Here we ignore the start-up period of the first $510^{4}$ slots to allow the system to reach steady-state. The gray line corresponds to the case $T=6, \lambda_{1}=\lambda, \lambda_{2}=1$, and $f_{1}=f_{2}=s_{1}=s_{2}=1$, whereas the black line corresponds to the case $T=3, \lambda_{1}=\lambda_{2}=\lambda, f_{1}=f_{2}=1$ and $s_{1}=s_{2}=2$, both plotted as functions of $\lambda$. (The confidence intervals for both curves are so tight that they are not visible.) We observe that the coordinated policy yields better backlog properties in the former case (gray line), while providing poorer backlog properties in the latter case (black line).

Indeed, the coordinated policy has some advantages in exploiting future service information of the slow resource for better decision making on the fast resource. This additional information, however, is imperfect because it does not take into account future arrivals, thus possibly providing a skewed perception of future queue lengths. As a result, even though a node may have a high backlog, it could be ill-advised to allocate the fast resource to this node because the node may not receive new customers and could be better served by the slow resource in the next cycle. Hence, coordination can actually hurt performance in some cases and neither of the two scheduling policies is strictly better than the other. It is worth pointing out that the relative improvement of each of the scheduling policies in Fig. 2 is relatively small, likely due in part to the fact that we only consider a small network instance.

The analysis presented in this paper is only a first step towards investigating the unique challenges posed by scheduling across different timescales. Further analysis is necessary to gain a better 


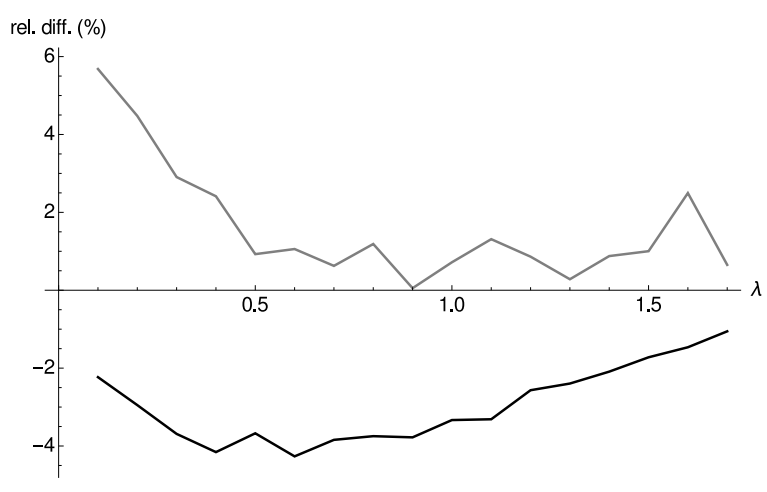

Fig. 2. The relative improvement of coordinated over uncoordinated scheduling for two stochastic networks with $N=2$ nodes.

understanding of these stochastic networks and explore the properties of coordination. In this case we can exploit existing results in the max-weight literature without timescale differences, such as handling, with more resources, time-dependent arrivals and feasible service rates [4], and intermediate nodes [13].

Although one strength of the max-weight algorithm is that it does not require knowledge of future arrivals for stability, a worthwhile extension is to modify the max-weight scheduling variants presented herein to learn and take into account the pernode arrival processes in order to better coordinate the various server resources. A related approach that includes future arrivals is based on Markov decision processes to determine the optimal policy; see, e.g., [10]. To the extent possible for large, real-life networks, this approach may help to derive structural properties and gain crucial insights into what constitutes a good scheduling policy, and in what settings coordination works well and provides the best performance.
Finally, it is worth noting that as $T \rightarrow \infty$, we obtain a system where the timescales are fully separated, and thus from the viewpoint of scheduling the fast resource it is as if the slow resource is fixed indefinitively. Here we may exploit existing results on stochastic networks with this form of timescale separation, such as so-called bandwidth-sharing networks [11].

\section{References}

[1] A.I. Bejan, R.J. Gibbens, Y. Lim, D. Towsley, A performance analysis study of multipath routing in a hybrid network with mobile users, in: Proc. ITC 25, 2013.

[2] H. Cao, et al., OnTheMark: Integrated stochastic resource planning of human capital supply chains, Interfaces 41 (5) (2011) 414-435.

[3] G. Celik, S.C. Borst, P.A. Whiting, E. Modiano, Dynamic scheduling with reconfiguration delays, Queueing Syst. 83 (1-2) (2016) 87-129.

[4] A. Eryilmaz, R. Srikant, J.R. Perkins, Stable scheduling policies for fading wireless channels, IEEE/ACM Trans. Netw. 13 (2) (2005) 411-424.

[5] L. Georgiadis, M.J. Neely, L. Tassiulas, Resource allocation and cross-layer control in wireless networks, Found. Trends Netw. 1 (1) (2006) 1-144.

[6] G. Giambene (Ed.), Resource Management in Satellite Networks, 2007.

[7] M. He, S. Murugesan, J. Zhang, Multiple timescale dispatch and scheduling for stochastic reliability in smart grids with wind generation integration, in: Proc. Mini-Conf. at INFOCOM, 2011, pp. 461-465.

[8] M. Khojastepour, A. Sabharwal, B. Aazhang, Information theory at multiple time-scales: the case of scheduling, in: Proc. Inl. Symp. Control, Comm. and Signal Proc., 2004, pp. 591-594.

[9] S. Kota, et al., Satellite component of NGN: Integrated and hybrid networks, Int. J. Satell. Commun. Netw. 29 (2011) 191-208.

[10] Y. Lu, S. Maguluri, M. Squillante, T. Suk, Delay-optimal scheduling for some input-queued switches, Perform. Eval. Rev. 44 (2) (2016).

[11] L. Massoulié, J. Roberts, Bandwidth sharing: objectives and algorithms, in: Proc. INFOCOM'99, 1999, pp. 1395-1403.

[12] M.J. Neely, Delay analysis for max weight opportunistic scheduling in wireless networks, IEEE Trans. Automat. Control 54 (9) (2009) 2137-2150.

[13] L. Tassiulas, A. Ephremides, Dynamic server allocation to parallel queues with randomly varying connectivity, IEEE Trans. Inf. Theory 39 (2) (1993) 466-478.

[14] A. Whittemore, S. Saunders, Optimal inventory under stochastic demand with two supply options, SIAM J. Appl. Math. 32 (2) (1977) 293-305.

[15] H. Xiao, H. Shao, K. Yang, F. Yang, W. Wang, Multiple timescale energy scheduling for wireless communication with energy harvesting devices, Radio-Eng. 21 (3) (2012) 815-822. 